\title{
Neuronal network analysis of serum electrophoresis
}

\author{
MAA Kratzer, B Ivandic, A Fateh-Moghadam
}

\begin{abstract}
Aims: To devise a system of neuronal networks which can classify the densitometric patterns of serum electrophoresis.

Methods: Digitised data containing 83 normal and 132 pathological serum protein electrophoresis patterns were presented to four neuronal networks containing 1900 neurons. Network 1 evaluates the integrated values of the albumin, $a 1, \alpha 2, \beta$ and $\gamma$ fractions together with total protein (Biuret method). Networks 2, 3, and 4 analyse the shape of the albumin, $\beta$ and $\gamma$ fractions. To increase the sensitivity for the detection of monoclonal gammopathies a Fourier transformation was applied to the $\beta$ and $\gamma$ fractions.

Results: After a learning period of 20 minutes (back-propagation learning algorithm) the system was tested with a set of electrophoresis patterns comprising 446 routinely collected samples. It differentiated between physiological and pathological curves with a sensitivity of $97.5 \%$ and a specificity of $98.8 \%$, with $86 \%$ correct diagnoses. All monoclonal gammopathies were recognised by the Fourier detector.

Conclusions: Neuronal networks could be useful for certain medical uses. Unlike rule based systems, neuronal networks do not have to be programmed but have the capacity to "learn" quickly.
\end{abstract}

The amount of medical knowledge needed to optimise treatment has increased over the past decades and is still growing. A physician can only know so much, so a knowledge deficit inevitably arises. Although specialisation has partly compensated for this, misdiagnoses and mistreatment occur when the appropriate expert is not available, or if cooperation between experts is limited. One way of improving this situation might be the application of so-called "expert systems". These are special computer programs that support physicians by emulating medical knowledge with artificial intelligence techniques.

In recent years considerable efforts have been made to develop such intelligence systems on the basis of the "If . . Then . . Else" rule. ${ }^{12}$ These can be realised with the declarative computer language PROLOG, which applies rules on a knowledge basis. If confronted with a question the PROLOG interpreter can find the algorithm to its solu- tion by itself.

Even if such systems could be used successfully in some limited and logically well structured medical areas, such as evaluation of pulmonary function tests, ${ }^{3}$ or cytological diagnosis of breast aspirates, ${ }^{4}$ the initial enthusiasm for using true artificial intelligence seems to have decreased. Several factors may be responsible.

1 Physicians tend to recognise intuitively similar patterns, so they are often not able to explain their decisions in terms of "If . . Then . . Else" rules. It is therefore difficult and time consuming for a "knowledge engineer" to extract such rules from a medical expert.

2 With increasing numbers of rules, or after updating the system with new rules, the probability that rules will interact unpredictably rises exponentionally, causing the system to misjudge.

3 Consequently, the physician is unable to understand the expert system and refuses to use its conclusions as the basis of a decision.

There is renewed hope that real world problems can be solved economically, using a new technology which aims to simulate biological nervous systems. These "intelligent" computer programs emulate the function of a network of artificial neurons on a conventional von Neuman computer. One considerable advantage of a neuronal network is its ability to "learn" (adjusting memory weights and thresholds by experience), thereby obviating the need for expensive and elaborate programming.

To investigate the potential of this new technology with respect to medical uses, we trained artificial neuronal networks to identify physiological and pathological curves of serum electrophoresis and compared the conclusions of those systems with the reasoning of a medical expert.

\section{Methods}

Trained artificial neuronal networks correlate inputs - that is, pathological electrophoresisto defined outputs - that is, monoclonal gammopathy. We used a network model with three layers of neurons, input, hidden and output cells (fig 1). The potentials of the input vector at the afferent line (example: input neuron 1, a1) of the input neurons (I1 ..n) are distributed via the potentiometers $(\mathrm{P} 1 \ldots \mathrm{P})$ to the afferent lines of all hidden neurons (H1 . . m). Hidden and output neurons transform the sum of all inputs using the mathematical sigmoid function $f(x)=1 / 1+\exp (-x)$ ). 


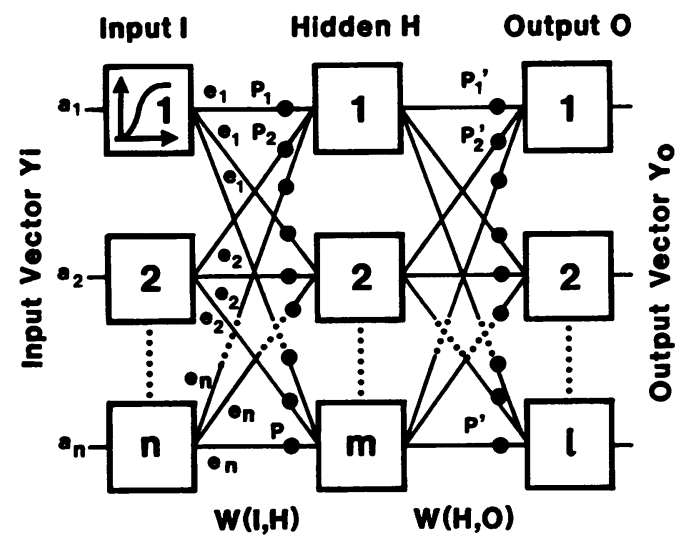

Figure 1 Schematic representation of the artificial neuronal network with three layers of neurons (input I, hidden $H$, Output $O)$. The input vector yi is imaged on to the afferent part (a1 . an) of the input neurons (1 . n). Hidden and output neurons apply the same mathematical sigmoid transfer function (example: inset of neuron 1). The efferent potential (e1 . en) is weighted via the potentiometers (p1 . p 7; weights are stored in the weight matrix $W(I, H))$ and applied to the afferent part of the hidden neurons $(1 \ldots m)$, where they are algebraically summed. Hidden and output layers are connected in an analogous way to yield the output vector yo.

They are connected in a similar way to the input and hidden layer. The resistances of all potentiometers, representing synapses with different strengths, are stored in the weight matrixes $\mathrm{W}(\mathrm{I}, \mathrm{H})$ and $\mathrm{W}(\mathrm{H}, \mathrm{O})$, respectively. Every neuron is also connected via a potentiometer ( $\left.P^{\prime \prime}\right)$ with the weight matrix $W(B)$ to a neuron with a constant potential of one unit (bias cells not shown).

TRAINING OF THE NETWORK

Learning of the neuronal network involves adjusting the weights $(\mathrm{W}(\mathrm{I}, \mathrm{H}), \mathrm{W}(\mathrm{H}, \mathrm{O})$ $W(B)$ ), initially present to random numbers, to generate the desired input/output relation for the training data. The back-propagation learning algorithm was used. ${ }^{5}$ For a given input vector (yi) of the training set the actual output (yo) is compared with the desired output. The error signal $E$ is then defined as:

$$
\mathrm{E}=\left\langle\left(\mathrm{y}_{\mathrm{o}}-\mathrm{y}_{\mathrm{o}}^{\mathrm{D}}\right)^{2}\right\rangle
$$

The angle brackets represent an overall average of the input patterns and output units. The use of a gradient descent technique for adjusting the weights, to minimise error, leads to the expression:

$$
\begin{gathered}
d W(H, O)=-e \frac{\delta E}{\delta W(H, O)} \\
=-2 e\left\langle\left(y_{o}-y_{o}{ }^{D}\right) \frac{\delta y_{o}}{\delta W(H, O)}\right\rangle
\end{gathered}
$$

where $\delta \mathrm{W}(\mathrm{H}, \mathrm{O})$ is the weight adjustment and e a selected adjustment rate. The $\delta \mathrm{W}(\mathrm{I}, \mathrm{H})$ and $\delta \mathrm{W}(\mathrm{B})$ is then calculated in an analog way.

\section{PATTERN IDENTIFICATION}

The complete information of a trained neuronal network is exclusively stored in the weights $\mathrm{W}()$ of the potentiometers. To classify an unknown electrophoresis curve, its pattern is presented to the input neurons. If the potential at any of the output cells overcomes a

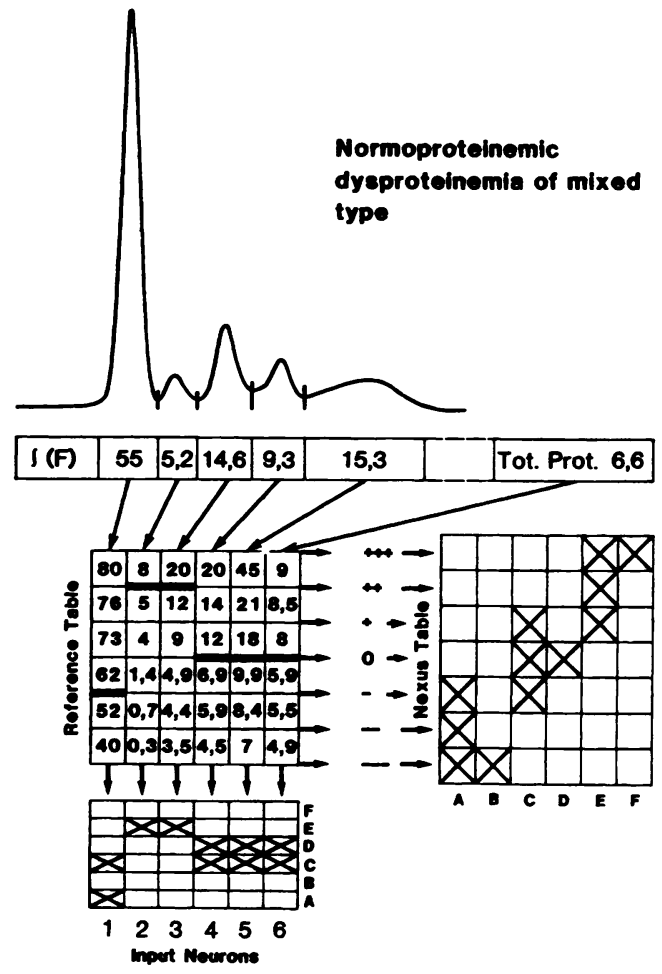

Figure 2 Preprocessing of serum electrophoresis densitograms for network 1. Digitised data received online from the densitometer corresponding to the heights of the electrophoresis curve were algebraically summed for every fraction (albumin, $\alpha 1$ and $2, \beta$ and $\gamma$ fraction). Depending on its position in relation to the reference values (Reference Table), the fraction was classified as $0,+$, $++\ldots$ These reduced data were imaged on an array of input neurons in a way defined by the Nexus table.

selected threshold the pattern is identified. For training and identifying we applied the network simulator Neurosoft (KI-Technik, Schmitz, Regensburg) on an IBM compatible computer (Escom AT12 MHz) with an 80286 processor, coprocessor and $1 \mathrm{MB}$ RAM.

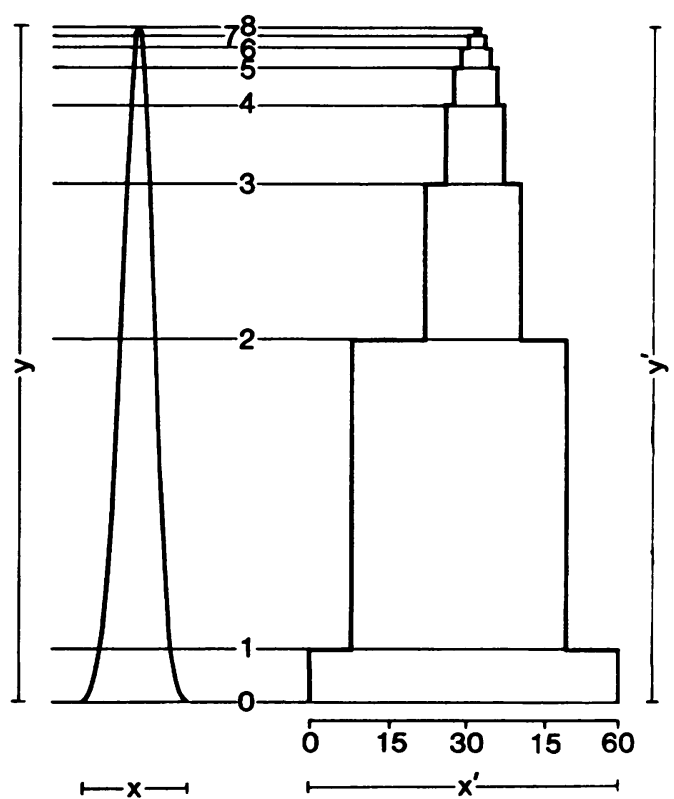

Figure 3 Preprocessing of serum electrophoresis densitograms for networks 2, 3, and 4. Integers $y$ of the fractions albumin, $\beta$ and $\gamma$ received online from the densitometer were normalised to a height of 8 and transformed to $y^{\prime}$. This reduced information was them imaged on to an array of $60 \times 8$ input neurons. 


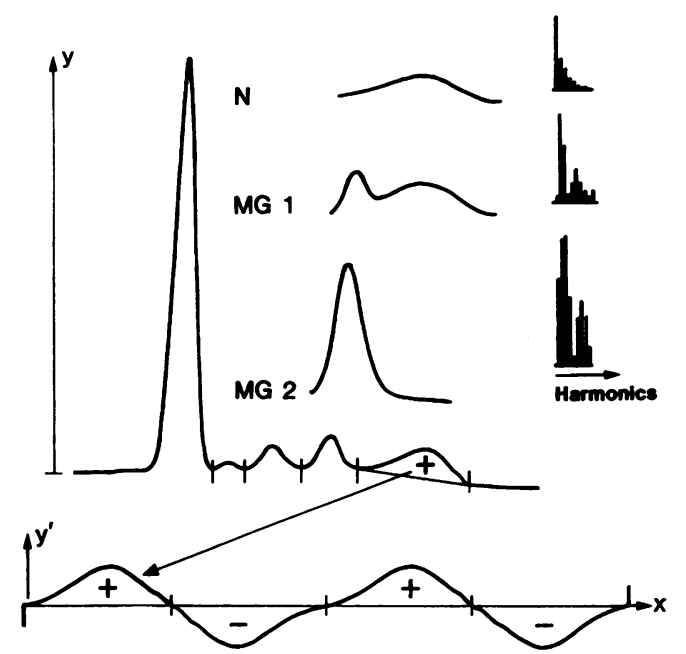

Figure 4 The $\gamma$ fraction of serum electrophoresis densitograms was mirrored at the $x$ axis and the Fourier transformation applied on the resulting oscillation (inset).

PREPROCESSING OF THE ELECTROPHORESIS DATA Protein electrophoresis was run on an Olympus Hite System 600 and the digitised data (12 Bit) were transferred via a RS 232 connection online to the computer. We preprocessed the data in three different ways and presented them to four different networks, each specialised in certain aspects of the electrophoresis curve.

Evaluating the integral of the curve (network 1) The albumin, $a 1, a 2, \beta$ and $\gamma$ fractions were integrated (fig 2) and expressed as a percentage of the integral sum. As further information, total protein (Biuret method) was added. According to its position in the Reference Table, data of every fraction are reduced to seven levels: normal $(0)$, three high $(+,++$, $+++)$, and three low $(-,--,---)$ scores. The Nexus Table describes which of the six

Table 1 Output diagnoses used

\begin{tabular}{lrl}
\hline Case No & $n=$ & \\
\hline 00 & $19(1)$, & $17(2), 18(3), 29$ (4) Normal pattern \\
01 & $8(1)$ & Artifical increase of albumin \\
05 & - & Hypoalbuminemia \\
02 & $3(2)$ & Anodic widening of the albumin fraction \\
03 & $1(2)$ & Biphasic albumin fraction (slow type) \\
04 & - & Biphasic albumin fraction (fast type) \\
31 & $1(1)$ & Increased $a-1$ fraction, acute, inflammation \\
25 & $5(1)$ & Hypoproteinemic dysproteinemia of mixed type \\
26 & $10(1)$ & Normoproteinemic dysproteinemia of mixed type \\
30 & $3(1)$ & Mild inflammatory $a$-dysproteinemic pattern \\
06 & $6(1)$ & An $a 1$ globulinemia? \\
08 & $3(1)$ & Increased $a 2$ fraction, \\
07 & $4(1)$ & Decreased $a 2$ fraction, diminished haptoglobin? \\
09 & $17(1)$ & Dysproteinemia of $a$ type \\
10 & $1(1)$ & Hypoproteinemia of $a 2 / \beta$ type, nephrotic pattern \\
11 & $1(1)$ & Normoproteinemic dysproteinemia of $\beta$ type \\
12 & $4(1)$ & Increased $\beta$ fraction, transferrin increase? \\
13 & $3(1)$ & Decreased transferrin? \\
18 & $14(1)$ & Polyclonal gammopathy \\
16 & $2(1)$ & Hyperproteinemic polyclonal hypergammaglobulinemia \\
17 & $2(1)$ & Polyclonal hypergammaglobulinemia, decreased transferrin? \\
20 & $8(3)$ & Polyclonal gammopathy with $\beta / \gamma$ bridge \\
14 & $8(1)$ & Hypogammaglobulinemic pattern \\
32 & $2(1)$ & Hypogammaglobulinemia and $a$-dysproteinemia \\
15 & $12(1)$ & 2 (4) Agammaglobulinemia \\
19 & $1(4)$ & $\beta / \gamma$-bridge, increase of Ig A? \\
21 & $7(4)$ & Suspicion of monoclonal gammopathy \\
22 & $4(3)$ & 21 (4) Monoclonal gammopathy \\
\hline & &
\end{tabular}

$\mathrm{N}=$ diagnosis number, $\mathrm{n}=$ number of pattern of the training set, $\mathrm{O}$ network number. input neurons ( $A, B, C, D, E, F)$ of the network 1 (144 input, 18 hidden, and 21 output neurons) for the six fractions are activated.

Evaluation of the shape of the curve (networks 2, 3, 4)

Three neuronal networks $(2,3,4)$ analyse the shape of the albumin, $\beta$ and $\gamma$ fractions. The peak of the curve is normalised to a length of 8 units, the $x y$ values are transformed to $x^{\prime} y^{\prime}$ according to fig 3 , and the data imaged on to an input array of $60 \star 8$ cells of a network with 10 hidden and 5 output neurons.

\section{Fourier transformation}

To increase the sensitivity for the detection of monoclonal gammopathies, the $\beta$ and $\gamma$ fractions $(+)$ were mirrored at the $x$ axis $(-)$ and a Fourier transformation applied on the resulting oscillation (fig 4). For a physiological $\gamma$ fraction curve this transformation has the great advantage of not only reducing the bias, but also eliminating high frequencies generated at the beginning and end of the curve. A monoclonal peak can be considered a high frequency superimposed on the low basic frequency of the polyclonal wave. The inset shows three examples of the harmonic distribution for a normal $(\mathbf{N})$ and two monoclonal $\gamma$ fractions (MG1, MG2).

\section{Controller}

If there is disagreement between the different networks on the diagnosis of a specific pattern, a controller instance decides which conclusion has to be made using an "If Or" inference mechanism.

\section{Results}

TRAINING

Table 1 lists the 28 output diagnoses and the corresponding number of typical electrophoresis patterns of the learning set (83 normal and 132 pathological), selected by a specialist, the type of network being shown in parentheses. The curves were presented to the system in a random sequence, the "tolerance" (output needed to classify a diagnosis as learned) was set to 850 and the "learning constant" $e(I, H)$ and $\mathrm{e}(\mathrm{H}, \mathrm{O})$ adjusted to 1 . The backpropagation algorithm showed a good convergence, and training of the largest network 1 took less than 30 minutes and about 400 repetitions of the whole training set.

\section{RECOGNISING}

The test set of electrophoresis patterns comprised 446 samples which were collected routinely on three different days. "Tolerance" was set at 800 . The system could differentiate between physiological and pathological curves with a sensitivity of $\mathbf{9 7 . 5 \%}$ and a specificity of $\mathbf{9 8 . 8 \%}$, with $86 \%$ correct diagnoses (tables 2 and 3). Significant errors occurred only in diagnoses $(8,10,15,20)$, which were trained with a small number (one to five) of patterns. All monoclonal gammopathies were recognised by the Fourier detector. 
Table 2 Differentiation between physiological and pathological electrophoreses

\begin{tabular}{lccc}
\hline & Normal & Pathological & Total \\
\hline Normal & 320 & 3 & 323 \\
Pathological & 4 & 119 & 123 \\
Total & 324 & 122 & 446 \\
\hline
\end{tabular}

Table 3 Recognition of the type of pathological electrophoreses

\begin{tabular}{lrr}
\hline Correct & 105 & $86 \%$ \\
Wrong & 17 & $14 \%$ \\
Total & 122 & $100 \%$ \\
\hline
\end{tabular}

\section{Discussion}

We have shown that a system of neuronal networks can be used to deal with a typical medical problem. ${ }^{6}$ Following a relatively short training period of about 20 minutes, using 215 selected typical electrophoresis patterns associated with 28 diagnoses, the system was able to process one new electrophoresis pattern every half second. It detected patterns indicative of disease with a sensitivity of $97.5 \%$ and a specificity of $98.8 \%$. The system correctly identified pathological electrophoreses with a probability of $86 \%$. The artificial network showed some weakness in diagnoses $8,10,15$ and 20 . The diagnostic capacity could be improved either by increasing the number of examples of the training set, or by increasing the neurons in the hidden layer, resulting in an improvement of the internal representation feature of the network.

However, the performance of the system might be better than the efficiency of a medical student after a similar training period. It was surprising how closely the artificial neuronal network imitated the behaviour of our expert in serum electrophoresis. In this context, it should be noted that this result was possible with a relatively small network comprising only 1900 neurons, which is the quantity of neurons in only six segmental ganglia of the leech Hirudo medicinalis. Again, we emphasise that our system was not programmed by an expert using a complicated set of rules. The network itself found the solution of adequately associating input/output relations by adjusting the interneural weights, using the back- propagation learning algorithm. This substantially reduces the developmental times of such systems.

A major advantage of a neuronal network is its ability to check the training data on consistency. If two equal input patterns are assigned to different output cells the algorithm cannot find a solution and the system will refuse to learn this pair. An additional interesting feature, especially for medical use, is the natural "fuzzy logic behaviour" of neuronal networks with the advantage of self defining the membership function. Even if the input vector is somewhat different, compared with the learned one, the program will respond with the most likely answer. Frick $e t$ al have shown the advantage of fuzzy logic in a rule based system for the diagnosis of bleeding disorders. (Conference proceedings, TLM Expositions, Liphook, England 1986.)

This capacity is closely connected to the ability of neuronal networks to process incomplete information and to generalise. When challenged with a unlearned pattern type, it will find the most appropriate answer. For instance, if trained to recognise horizontal and vertical bars and confronted with a diagonal one, the system will respond reasonably well with a horizontal and vertical output, but both cells will have a reduced potential.

To use neuronal networks effectively adequate preprocessing with the aim of reducing data is essential. Otherwise the training set becomes too large and learning time increases exponentially. In our model we have tried to imitate the physician who usually checks the integrated fractions, and in a second step, looks at the shape of the curve.

1 Knüppel W, Neumeier D, Fateh-Moghadam A, Knedel M. Rechnerunterstützte Befundung von Eiweißelektrophoresen auf Celluloseacetatfolie. $\mathcal{f}$ Clin Chem Clin Biochem 1984;22:407-17.

2 Shortliffe EH. Computer-based medical consultations: Mycin. New York: Elsevier, 1976.

3 Kunz JC, Fallat RJ, McClung DH, et al. A physiological rule based system for interpreting pulmonary function test results. Proc Comp Crit Pulmonary Med 1979:375-9.

4 Heathfield H, Bose D, Kirkham N. Knowledge-based computer system to aid in the histopathological diagnosis
of breast disease. $\mathcal{F}$ Clin Pathol 1991;44:502-8.

5 Rumelhart DE, Hinton GE, Williams RJ. Learning internal representation by error propagation. In: Rumelhart DE, representation by error propagation. In: Rumelhart DE, McClelland JL, eds. Parallel distributed processing. Vol. 1.
Foundation. Cambridge. MA: MIT Press 1986:318-62.

6 Foundation. Cambridge. MA: MIT Press 1986:318-62. analysis of serial cardiac enzyme data. Am $\mathcal{f}$ Clin Pathol 1991;96:134-41. 